\title{
Biomechanics in bone tissue engineering
}

\author{
Dominique P. Pioletti* \\ Laboratory of Biomechanical Orthopedics, EPFL, Station 15, 1015 Lausanne, Switzerland
}

(Received 15 September 2009; final version received 18 January 2010)

\begin{abstract}
Biomechanics may be considered as central in the development of bone tissue engineering. The initial mechanical aspects are essential to the outcome of a functional tissue engineering approach; so are aspects of interface micromotion, bone ingrowths inside the scaffold and finally, the mechanical integrity of the scaffold during its degradation. A proposed view is presented herein on how biomechanical aspects can be synthesised and where future developments are needed. In particular, a distinction is made between the mechanical and the mechanotransductional aspects of bone tissue engineering: the former could be related to osteoconduction, while the latter may be correlated to the osteoinductive properties of the scaffold. This distinction allows biomechanicians to follow a strategy in the development of a scaffold having not only mechanical targets but also incorporating some mechanotransduction principles.
\end{abstract}

Keywords: bone scaffold; load-bearing application; biomechanics; mechanotransduction; tissue engineering

\section{Introduction}

\section{Bone tissue engineering in short}

The justification for developing a bone scaffold is usually motivated by the fact that the gold standard for bone substitutes is still the autograft despite the invasive, traumatic procedure it requires and the limited amount of bone that can be obtained. As most of the researchers in this field have no surgical background to adequately appreciate exactly how invasive such a procedure truly is, we report, in Figure 1(a), the particular case of a posterior surgical approach to the iliac crest to underscore what 'traumatic' really means during the collection process of an autograft. Moreover, as can be seen in Figure 1(b), the volume of the obtained bone graft is limited. The low yield as well as the aggressiveness of such a procedure motivates the need to develop alternatives to autografts for bone reconstruction. Bone tissue engineering is an attractive approach, providing its results match those obtained with autografts. Bone tissue engineering consists of the combination between a scaffold for the osteoconductive aspect, cells and/or growth factors for the osteoinductive aspect and biomechanics as most of the treated sites are load bearing. In this paper, the aspects of biomechanics will be reviewed, and it will be shown that osteoinductive properties may also be harnessed from the mechanical stimulation of cells resulting from scaffold deformation.

\section{Bone tissue engineering offers a new field for biomechanics}

Traditionally, biomechanical investigations have heavily focused on bones and orthopaedic implants. The knowledge acquired in these applications can be applied and extended to the field of bone tissue engineering, as it combines aspects of both bone and implant biomechanics. Moreover, as noted by Meyer et al. (2004), biomechanical considerations have not been completely integrated into the development of bone tissue engineering solutions. The need for biomechanical studies is clearly justified when we consider, for instance, that the absence of mechanical loading could affect the osteoconduction of well-accepted biomaterials such as calcium phosphate granules (Handschel et al. 2002). Indeed, bone tissue engineering constructs promote more bone repair when dynamic loading is applied (Guldberg 2002). Biomechanical aspects of bone tissue engineering have therefore become as important as biomaterial considerations.

From a general point of view, two aspects have been considered with biomechanical studies in bone tissue engineering: structural biomechanics and mechanotransduction. The former is related to the osteoconductive properties of the scaffold, while the latter concerns its potential osteoinductive properties.

\section{Different applications may require different scaffold biomechanics}

Applications for bone reconstruction often require scaffolds with a specific set of mechanical properties, which may vary greatly from one case to another, as can be seen in Table 1. Revision procedures in total hip arthroplasty, tibial or femoral osteotomy and maxillofacial situations are the most demanding in terms of scaffold biomechanics. In other applications reported in Table 1,

\footnotetext{
*Email: dominique.pioletti@epfl.ch

ISSN 1025-5842 print/ISSN 1476-8259 online

(C) 2010 Taylor \& Francis

DOI: $10.1080 / 10255841003630660$

http://www.informaworld.com
} 

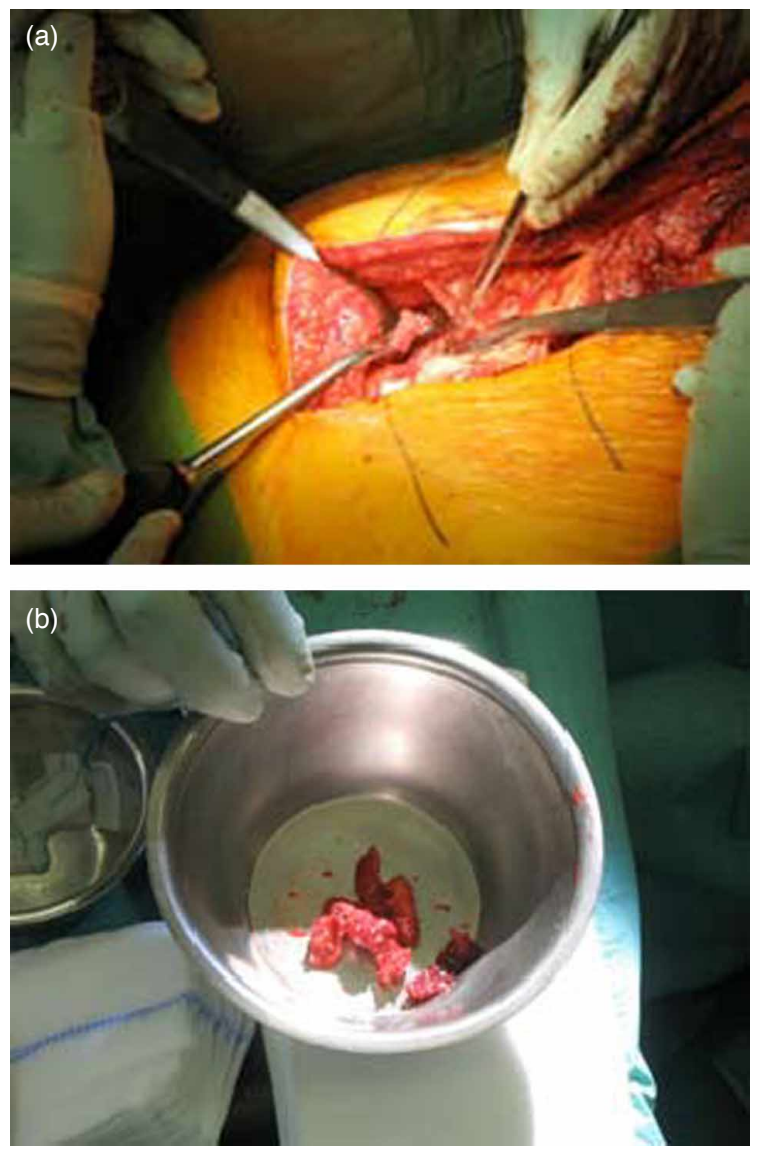

Figure 1. (a) Posterior surgical approach to the iliac crest. (b) Total amount of autograft obtained during the procedure (courtesy of Dr Constantin Schizas from the Orthopedic Hospital, CHUV, Lausanne, Switzerland).

the load could be distributed between the scaffold and the implant or the external fixation. The required volume of the bone substitute varies between different applications; the largest defects usually arise following massive tumour resections. Important volumes may also be needed in osteotomies. It is important to note that the access to the iliac crest may not always be possible in certain surgical procedures involving traumas, for instance. For maxillofacial surgery, the access to the iliac crest necessitates different surgical facilities and anaesthesia procedure from those commonly used for maxillofacial surgery. For all applications needing a bone graft taken from the iliac crest and especially for maxillofacial applications, the graft collection is then an additional, heavy surgical procedure which, reports show, has nonnegligible associated morbidity (Silber et al. 2003).

In musculoskeletal applications, bone scaffolds need to have carefully defined biomechanical properties so as to use them in situations where they can function immediately following their surgical insertion. This requirement is at the origin of a new approach called 'functional tissue engineering', coined as such as early as 1998 during a meeting of the American Society of Biomechanics. One emphasis of functional tissue engineering is 'to identify the critical structural and mechanical requirements needed for each tissue engineered construct' (Butler et al. 2000). Moreover, for bone tissue engineering, the most important design parameter is to allow the scaffold an effective remodelling/adaptation to physical demand. We see then, as already mentioned, that biomechanics in the development of a bone scaffold has so far mainly focused on mechanical aspects and only partially on mechanotransduction.

\section{Mechanical aspects for bone tissue engineering Bone mechanics}

The definition put forth by Juncosa et al. (2003) regarding functional tissue engineering states that 'functional tissue engineering seeks to regenerate damaged tissue by first measuring in vivo forces and/or strains transmitted by the normal tissue and then using these results to develop design criteria so that engineered constructs can effectively resist these loading regimes after surgery'. In a first step, bone mechanical properties should indeed be well defined. This includes morphological aspects such as bone permeability which directly affects interstitial fluid flow (Tate and Knothe 2000), bone anisotropy which is considered either transverse isotropic or orthotropic

Table 1. Different applications for bone grafts.

\begin{tabular}{|c|c|c|c|}
\hline Surgical applications & Mech. prop. & Volume & Access iliac crest \\
\hline Spinal fusion & $+1-$ & $+1-$ & + \\
\hline TKA revision & + & + & $+1-$ \\
\hline THA revision & ++ & + & + \\
\hline Trauma & $+1-$ & $+1-$ & $+1-$ \\
\hline Osteotomy & ++ & ++ & + \\
\hline Maxillofacial & ++ & $+1-$ & -- \\
\hline Tumour & + & +++ & $+1-$ \\
\hline
\end{tabular}

Notes: For these applications, an evaluation of the mechanical properties, volume and access to iliac crest is given. + indicates either high mechanical properties, high volume or easy access to iliac crest while - represents the opposite. \pm corresponds to an average situation. 
(Cowin 2001) and hierarchical tissue organisation which is described through a multi-scale approach (Ghanbari and Naghdabadi 2009). As it is not the goal of this review to report in detail this kind of information, interested readers should refer to the numerous literature listings already existing in this field and in particular to those focusing on bone tissue engineering applications (e.g. Athanasiou et al. 2000).

\section{Loading conditions}

The material properties of bone are but one part of the mechanical aspects to be taken into account. It is obvious that the particular loading condition also needs to be evaluated. In the case of tibial osteotomy, for example, a biomechanical analysis was performed to define mechanical targets for scaffold development (Blecha et al. 2005). These targets were partially reached by developing a composite scaffold made of polylactic acid reinforced with micrometre calcium phosphate particles (Mathieu et al. 2006). Many different scaffolds have been developed for bone tissue engineering, but only a few have taken into consideration the mechanical aspect as a main design target. Usually, mechanical aspects are described only at the end of the development by quantifying the scaffold mechanical properties. In the development of a biodegradable scaffold, evaluation of how changes in the relative amount of porogen and calcium phosphate affect the strength of the material has been studied (Porter et al. 2000). As porosity plays a significant role in the mechanical properties of the scaffold, development of a design optimisation scheme for 3D internal scaffold architecture to match desired elastic properties and porosity has been simultaneously proposed (Lin et al. 2004). Indeed, highly interconnected porous scaffolds are needed to promote 3D tissue growth, nutrient diffusion and vascularisation. The matching of trabecular morphological and mechanical properties has then been a natural target, which has been reached using a technique based on supercritical $\mathrm{CO}_{2}$ foaming (Mathieu et al. 2005). As can be seen in Figure 2, the developed scaffold has morphological properties similar to trabecular bone, and its elastic compressive modulus at $75 \%$ porosity is around $160 \mathrm{MPa}$, close to the human bone trabecular compressive modulus. The next step in the mechanical characterisation of bone scaffolds will certainly need to account for their viscoelastic behaviour, mirroring what has been done for soft tissues (e.g. Pioletti and Rakotomanana 2000), especially if the scaffold is made of synthetic polymer.

\section{Interface biomechanical analysis}

The aspects of scaffold incorporation were also mentioned in the definition of functional tissue engineering
Trabecular bone (rod-like structure)

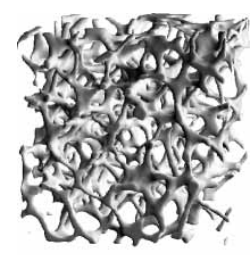

Biocomposite

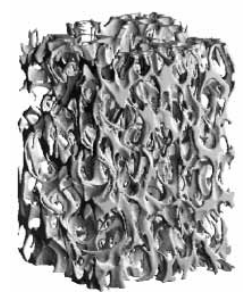

Figure 2. $\mu \mathrm{CT}$ images of trabecular bone presenting a rod-like morphology (top) and a biocomposite scaffold made of PLA and $\beta$-TCP particles similar to trabecular bone morphology. Different morphological structures (plate-like or hybrid-like) can be obtained simply by modifying physical parameters with a supercritical $\mathrm{CO}_{2}$ foaming technique (Mathieu et al. 2006).

(Butler et al. 2000). As usual for orthopaedic implants, the micromotions at the interface of the scaffold and the bone certainly play an important role in scaffold incorporation. Excessive micromotion at the interface may mechanically impair the osteointegration of the scaffold (Meyer et al. 2004). Indeed, for aspects of scaffold integration, Brunski (1991) proposed that biomechanics is more important than biomaterial properties per se.

Unlike metallic orthopaedic implants, which have an elastic modulus several orders of magnitude higher than bone, the amplitude of the micromotion at the interface of the scaffold and the bone is correlated to the scaffold's mechanical properties as well as to its size (Blecha et al. 2005). Depending on the values of the micromotions, bone resorption can be induced (Stadelmann et al. 2008) and a fibrous tissue may be produced around the scaffold (Jasty et al. 1997). Biomechanical analyses could also be used to anticipate the tissue differentiation based on the knowledge of the micromotion values (Prendergast et al. 1997; Carter et al. 1998; Buchler et al. 2003).

\section{Evolution of scaffold mechanical properties}

The major difference in the biomechanical analysis of conventional orthopaedic implants versus bone scaffolds is that the mechanical properties of the scaffold will change over time as its degradation occurs. The cornerstone of biodegradable bone scaffolds is that the decreased mechanical properties of the scaffold during its degradation will be compensated for by the increased mechanical properties of the new bone formation inside the scaffold (Behravesh et al. 1999). It would then be important to anticipate the degradation process with respect to the 
scaffold mechanical properties. We may imagine the following similar theoretical developments as those done with bone remodelling driven by biomechanical parameters (Carter et al. 1987; Huiskes et al. 2000), but applying this approach to scaffold remodelling. In this case, scaffold remodelling will mean bone ingrowths in the scaffold and scaffold degradation. Experimental data on scaffold degradation will then be necessary. Indeed, most synthetic biodegradable polymers degrade by passive hydrolysis. They undergo bulk degradation, which leads to a sudden drop in mechanical properties without a change in the overall dimensions of the polymer. It will then be difficult to evaluate its biomechanical properties solely based on imaging data. Moreover, depending on the scaffold composition, its degradation time can range from weeks to years (Behravesh et al. 1999). Obviously, only in vivo studies would be able to feed the parameters of a model for scaffold remodelling. Specific in vivo studies on the degradation aspect of bone scaffolds are almost nonexistent, and this point will certainly need to be further described in the future. Relevant in vivo models need to be developed, which brings another complexity to the study. Large animal models are usually preferred for biomechanically oriented questions, while small animals are preferred for biologically oriented questions (Liebschner 2004).

There is an interesting paradox in the development of scaffolds for tissue engineering in the sense that a lot of effort is invested in trying to mimic the morphological and mechanical properties of natural bone, while the final goal is that the scaffold will be degraded and completely replaced by natural bone. Despite how frustrating this may seem, the rationale for this approach is supported by some experimental data. For example, in the field of orthopaedic implants used as drug delivery systems, it has been demonstrated that the use of anti-resorptive drugs such as bisphosphonate coated on the implant increases the osteointegration of the implant and its mechanical stability (Peter et al. 2006). A possible explanation for such a result has been suggested by Aspenberg who described the effect of the anti-resorptive as a protection against early bone resorption following the insertion of an implant (Aspenberg 2009). The maintained bone then plays the role of a scaffold allowing new bone formation and remodelling. The artificial bone scaffold, with morphological and mechanical properties close to the natural bone, therefore, probably has the same role in supporting new bone formation before being resorbed. A completely different view also exists in the sense that during the healing of a tissue, the initial structures are different from normal tissue. It was then proposed to engineer scaffolds that promote tissue remodelling, instead of trying to mimic the final tissue form and function (Ingber et al. 2006). The knowledge gathered from developmental biology and systems biology could also be harnessed to better design tissue engineering applications (Lenas et al. 2009). Both approaches in the development of bone scaffolds are probably complementary, especially if the design of a functional tissue is the target.

\section{Computer methods for bone tissue engineering mechanics}

To tackle the mechanical aspects for bone tissue engineering, computer methods have been extensively used. One of the major questions addressed in these studies concerns the mechanical integrity of the scaffold used in loaded situations. For example, Brazel and Taylor (2009) extended a technique used to predict fatigue failure in metal and composites and applied it for bone graft material. The results showed that graft materials with much lower mechanical properties than cortical bone could sustain physiological loads due to a reduction in local stress concentration. Computational analysis could also be used to evaluate the mechanical properties of custom-made scaffolds and verify that targeted values are obtained (Williams et al. 2005). For an application in revision knee arthroplasty, Terrier et al. (2009) evaluated the impact of scaffold size on primary stability when used to fill bone defects in the tibial plateau. A more specific application lies in a proposed patient-based approach for replacing vertebral bodies with the help of computational methods as central tools using building blocks for the biomechanical evaluation of the developed scaffold (Wettergreen et al. 2005). A detailed biomechanical description of scaffolds can be obtained through a microcomputed tomography-based finite element model. With this approach, it is possible to evaluate the inhomogeneity of the stress and strain fields in a scaffold submitted to mechanical loading, which could be correlated to bone formation in specific scaffold locations (Sandino et al. 2008). Regarding the evolution of the scaffold and bone mechanical properties, an original computational approach evaluated the change in strain energy of bone scaffold systems during the bone regeneration process (Adachi et al. 2006). The optimal scaffold system was defined as the one with its microstructure, inducing a constant stiffness close to healthy bone over the entire scaffold degradation and bone regeneration process. This approach could then open the door to incorporating mechanotransductional considerations in scaffold development.

\section{Mechanotransduction aspects for bone tissue engineering}

\section{Mechanical effect on cells}

While it is clear that the mechanical aspects are essential for the clinical success of a bone scaffold, bone ingrowths in the scaffold are necessary, a factor that does not come 
into play for metallic implants. The 'remodelling' of the scaffold should finally lead to a complete healing of the treated bone. Bone ingrowth is driven by cells from the host. It is therefore evident that the effect of biomechanical stimuli on cells due to the scaffold deformation has to be taken into account. As mentioned by Sikavitsas et al. (2001), apart from the biochemical strategy which mostly relies on the addition of growth factors and the selection of ideal osteoconductive and biodegradable materials for the scaffold, mechanotransduction could be used to control the proliferation and differentiation of bone cells. This point has also been observed by Klein-Nulend et al. (2005) who stated that 'in bone tissue engineering, it is essential to understand how mechanical conditions affect the formation of bone matrix components by the cells at a local level in order to generate tissues which will be functionally appropriate'. Mechanotransduction aspects per se of bone cells have been well studied and some aspects are reported here.

\section{Mechanotransduction in bone cells}

Bone cells are known to be responsive to mechanical stimulation. For example, the effect of different regimes of fluid flow on bone cells has been studied, which demonstrated that mechanical stimulation plays an important role (Jacobs et al. 1998). In addition to effects obtained via fluid-mediated stimulation, stretching the surface on which the cells are attached can also induce bone cell differentiation (Cavalcant-Adam et al. 2002). This mechanical stimulus has been shown to be integrin mediated (Lacouture et al. 2002). These two examples highlight the two possible mechanisms - fluid flow and/or stretching of the cells through extra-cellular matrix deformation - by which bone cells may be mechanically stimulated. Different models have been developed to gain more information on the mechanotransduction aspect of bone, such as, for instance, the use of an analytical approach for osteocyte-level fluid flow induced in a bone (Srinivasan and Gross 2000). A specific model was proposed to explain the paradox between in vivo strain and in vitro mechanical experiments, which needed one order of magnitude higher strain to induce a biological response. Indeed, transverse tethering filaments were described to amplify small forces by transmitting from large structures to transducers (Han et al. 2004). When we want to interpret mechanotransduction results to target bone scaffold development, it is important to recall that cells do not sense the mathematical concepts of stress or strain but that instead, as proposed by Humphrey, we should focus on force aspects at the atomic or molecular level (Humphrey 2001). However, the concepts of stress and strain for translational characterisations have so far been the only ones that could be effectively used.

\section{Translation of mechanotransduction knowledge in the} development of bone scaffold

As for mechanotransduction studies, it has been proposed that fluid flow and its corresponding transport-induced process are important mechanical aspects to be considered in the development of bone scaffolds (Tate and Knothe 2000). Despite all these results, most of the gathered mechanotransduction knowledge has been used only for the development of bioreactors to optimise in vitro tissue formation in scaffolds (Bilodeau and Mantovani 2006). Rotating bioreactors have been designed to increase mass transfer by inducing dynamic flow conditions in culture (Yu et al. 2004). Fluid shear stress generated in a flow perfusion bioreactor was used as an osteoinductive factor on mesenchymal stromal cells (Datta et al. 2006). Other mechanical stimulations, such as strain induced by a scaffold subjected to a four-point bending, induced the osteogenic differentiation of mesenchymal stromal cells (Mauney et al. 2004). Rotating bioreactor and flow perfusion bioreactor approaches are therefore successful in enhancing the mineralisation of the formed matrix; however, no mechanical properties of the constructs have usually been reported. In a review of bone tissue engineering for maxillofacial surgery, it was reported that the early positive results obtained in rotating bioreactors are not always reproducible, suggesting the possibility that the non-physiological forces generated may damage the cells in the scaffolds (Wiesmann et al. 2004). It has also been reported that the conditions of the fluid flow in a bioreactor affect the pattern of bone formation in general (Meinel et al. 2004), particularly the osteogenic differentiation process in mesenchymal stromal cells (Stiehler et al. 2009). New designs, such as the biaxial rotating bioreactor (Zhang et al. 2009), are continuously developed and are mostly based on empirical approaches, with the exception of those designed based on theoretical developments (Singh et al. 2005) or the description of local strains sensed by the cells (Pfeiler et al. 2008). To increase the responsiveness of the mechanically stimulated cells in a bioreactor, mechanical stimulation was combined with drug supplementation, which has an effect in increasing the opening time of mechanosensitive voltage-operated calcium channel (Wood et al. 2006). A positive effect using this strategy was observed for the calcification of the osteoid.

As previously mentioned, the in vitro bioreactor approach to create functional bone tissue engineering has not yet been fully successful. A recent review by Martin et al. (2009) highlights the difficulty of obtaining consistent engineered tissue. With bioreactors for large scaffolds, the transport of nutrients must be provided through convection, and it is a challenge to find the optimal perfusion velocity ensuring, for example, an oxygen supply in the centre of the scaffold without inducing a 
detrimental shear stress on the membrane (Pierre and Oddou 2007). Nevertheless, a bioreactor with controlled mechanical stimuli is a very interesting in vitro system for studying mechanotransduction phenomena and could be correlated to numerical studies (e.g. Jungreuthmayer et al. 2009). Given the hurdles still facing in vitro bioreactor approaches, reconstructing large bone defects where the need for engineered bone is important may require alternative approaches such as using bone itself as a bioreactor to produce an engineered bone construct (Stevens et al. 2005). This concept has been demonstrated in a rabbit model where an artificial space between the tibia and the periosteum was created and filled with a hydrogel, thereby creating an in vivo bioreactor. This bioreactor yielded engineered bone presenting similar biomechanical properties compared to native bone. Despite being difficult to translate to a clinical application, the study of Stevens et al. at the very least demonstrated that the best bioreactor for bone scaffold is therefore probably bone itself with the idea that a scaffold could indeed mature into a normal bone tissue if an adequate environment is provided in vivo. Following this observation, it would then be advantageous to use mechanotransduction phenomena in vivo by designing a scaffold with mechanical properties allowing 'osteoinductive fluid flow' in the scaffold.

As mentioned by Guldberg (2002), 'an understanding of how mechanical signals affect construct integration and regeneration of function may provide microstructural design objectives for 3-D scaffold architectures and may have an impact on the selection of scaffold material, cell type or seeding density, and other construct parameters'. Based on this need, an analytical model has been developed allowing to optimise the fluid flow exchange between the scaffold and its surrounding, while maintaining the value of shear stress on cells within physiological ranges (Blecha et al. 2009). The advantages of the analytical approach reside in an effective parameter study, particularly determining the impact of the elastic modulus, Poisson's ratio, porosity and permeability of the scaffold on fluid motion, which is complementary to numerical studies (e.g. Byrne et al. 2007). There is probably an important potential in developing bone scaffolds by combining mechanical aspects and mechanotransduction knowledge in order to target simultaneously the mechanical integrity of the reconstructed bone defect and osteoinduction through the scaffold-mediated mechanical stimulation of cells.

\section{Computer methods for bone tissue engineering mechanotransduction}

Unlike the computer methods described above for mechanical aspects of bone tissue engineering where no consideration of the biological system is made, in this section the described computer methods clearly try to incorporate some mechanotransduction aspects in their description. For example, by combining 3D imaging and numerical simulation of scaffold physical properties, information on pore morphologies can be deduced to obtain bone ingrowth in bone scaffold (Jones et al. 2009). In particular, it was verified that a threshold permeability of $\sim 3 \times 10^{-11} \mathrm{~m}^{2}$ was necessary for inducing vascularisation and mineralisation in the bone graft. One of the most rational targets for a scaffold development would probably be mimicking the native bone strain. Following this idea, $\mu \mathrm{CT}$ measurement and FEM could be used to develop a scaffold for which the strain histograms for scaffold and native trabecular bone under the same loading conditions must be similar. Osteogenic loading conditions for bone tissue engineering should then be obtained (Jaecques et al. 2004). By combining finite element methods with computational fluid dynamics, it could be possible to optimise the level of compression and strain rate applied on a scaffold to favour osteogenesis (Milan et al. 2009). Computational models can also describe the large difference in cell wall shear stress induced by fluid flow either between different scaffolds or even in the same scaffold presenting an inhomogeneous distribution of pore diameters (Jungreuthmayer et al. 2009). The introduction of mechanotransduction aspects for bone tissue engineering clearly necessitates a multi-scale approach in order to transform the macromechanical stimulation at the level of the scaffold to a micromechanical stimulation acting at the cellular level, which will drive the biological response needed for adequate bone ingrowth. This multi-scale approach has been followed by Sanz-Herrera et al. (2008), and it was found that bone ingrowth at the scaffold surface was proportional to the cell concentration and regulated by the local strain energy. The multi-scale approach is indeed the only way to obtain an evaluation of cell deformation embedded in a scaffold as apparent from the work done, for example, on muscle tissue construct (Breuls et al. 2002). Another approach by Prendergast consists in applying a mechanoregulation algorithm, which drives the tissue differentiation by taking into account the mechanical properties of a scaffold for an osteochondral defect (Kelly and Prendergast 2006). This strategy was extended to bone tissue engineering and a new approach was introduced, which consisted in applying stochastic processes for describing cell proliferation and migration (Byrne et al. 2007). One of the major results showed that due to the different loading situations found in the body, scaffolds should be tailored specifically for each implantation site. This loading-specific approach in the development of scaffolds has been recently taken into account in the development of an algorithm optimising the fluid flow to maximise transport, while keeping the 
shear stress on cells within an osteogenic range (Blecha et al. 2009).

In addition to mechanotransduction aspects, other factors such as nutrient transport, cell adhesion and migration or cell-scaffold mechanical interaction have been considered in different computational models and were recently reviewed (Sengers et al. 2007). As mentioned by Lacroix et al. (2009), only a few computational models take into account biology when describing bone scaffolds. Finally, an important body of literature exists on computational aspect of bone healing taking into account mechanotransduction aspects (e.g. Epari et al. 2006). Although these are not biologically similar to the bone tissue engineering situation, some developments could still be adapted for the development of new bone scaffolds.

\section{Biomechanical paradigm in bone tissue engineering}

In summary, the biomechanical paradigm in bone tissue engineering could be split into four steps as described in Figure 3, which uses the particular example of a tibial osteotomy. In each of the four steps, the mechanical aspects are central to insure the safety of the surgical procedure using a bone scaffold. The mechanical aspects also induce, at each step, a biological reaction around and in the scaffold through the process of mechanotransduction. On one hand, the mechanical and mechanotransductional aspects are obviously linked in each step, while on the other hand the separation of the four steps follows a chronological process from a mechanical point of view. These separations between the four steps and between mechanics and mechanotransduction could be used as an engineering approach in the design of bone scaffolds, allowing the

Biomechanical paradigm in bone tissue engineering

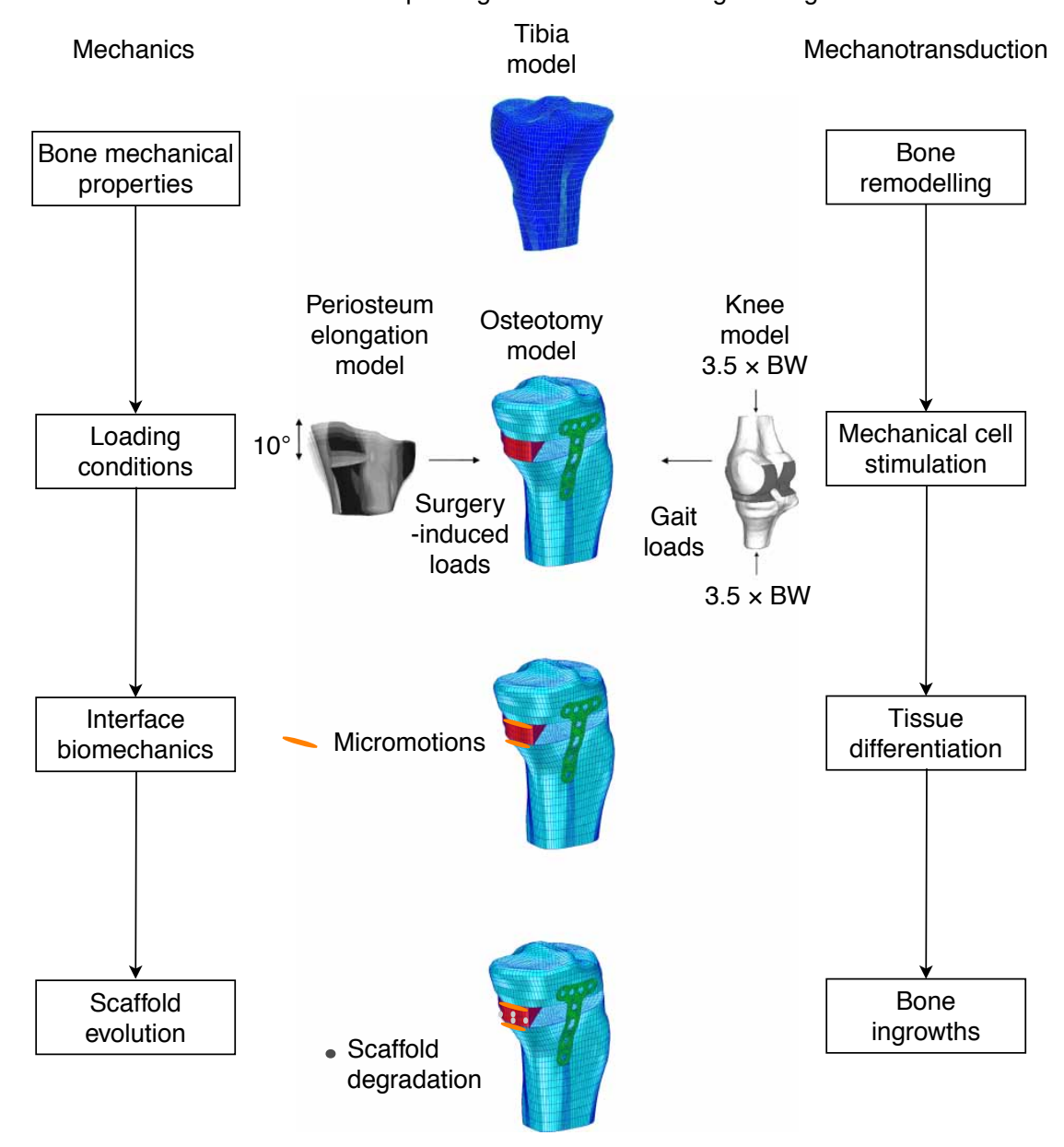

Figure 3. The mechanical aspects for bone tissue engineering are separated into four steps. The first two steps can be considered as the primary fixation of the scaffold by analogy to the terminology used in orthopaedic implant practice. The third step is comparable to a secondary fixation, while the fourth step introduces a new concept specific to biodegradable materials, which could be called 'final fixation'. Each step has a corresponding mechanotransduction aspect. 
discrimination between possible mechanical targets. For example, the degradation of the scaffold would also influence the interface biomechanics, which could in turn affect the loading transmission between the scaffold and the bone, therefore affecting bone ingrowth into the scaffold. A dynamic coupling then certainly exists between the four steps and between mechanics and mechanotransduction.

Paralleling the nomenclature used with non-cemented metallic orthopaedic implants, we could consider the first two steps (bone mechanical properties and loading conditions) analogous to the primary fixation of the scaffold, while the third step (interface biomechanics) would be identified as the secondary fixation. For the fourth step (scaffold evolution), we would need to introduce a new concept, namely 'final fixation'. At this final step, the mechanical support should be taken by the host bone.

\section{Future position of biomechanics in bone tissue engineering}

As our understanding of mechanotransduction phenomena in bone increases, it is clear that the next step for biomechanics in bone tissue engineering is to integrate more extensively this knowledge in the development of bone scaffolds (Butler et al. 2009). Mechanotransduction aspects could be considered in each of the four steps given in Figure 3. In the first and second steps, the so-called primary fixation, it is clear that the developed scaffold should not induce a stress-shielding effect, resulting in peri-scaffold bone resorption as seen for the metallic joint implants. The elastic property of the scaffold should therefore not exceed that of bone in order to maintain a proper mechanical stimulation on the peri-scaffold bone. This mechanical stimulation will also obviously be driven by the loading conditions given by the clinical situation considered in order to be osteogenic. Scaffolds should then ideally be developed for particular clinical situations corresponding to particular loading conditions as suggested recently (Byrne et al. 2007). In the third step, called the secondary fixation, mechanotransduction knowledge gathered from tissue differentiation around metallic implants could generate some applications. In particular, micromotions were shown to influence the tissue differentiation around metallic implants (Jasty et al. 1997). This effect has also been modelled (e.g. Buchler et al. 2003). The mechanical properties of the scaffold could then be adapted to generate interface scaffold/bone micromotions, inducing an osteointegration of the scaffold as proposed for a tibial osteotomy application (Blecha et al. 2005). In the fourth step, called the final fixation, the bone should support the mechanical load as the scaffold degrades. This is certainly the most difficult part in the scaffold design, as there is no clear target regarding the degradation rate for the scaffold. This load transfer between the scaffold and the bone could be appreciated in the context of fracture healing or foetal development, where this phenomenon of load transfer between different structures is happening. Mechanotransduction knowledge of these two situations could then find some applications in scaffold development as suggested by Lacroix et al. (2009).

In conclusion, the ideal situation would, through mechanical considerations, confer osteoinductivity to a synthetic bone scaffold in order to reduce the dependency of this material on osteogenic factors such as drugs or biological products. If the developed scaffold can in and of itself induce bone ingrowth, following the incorporation of mechanotransduction concepts in its development, an offthe-shelf product could be obtained. This approach would obviously avoid the burden of the regulatory affairs related to bioreactors (Martin et al. 2009) and facilitate the translation of the developed scaffold into clinical practice.

Mechanical aspects are therefore fundamentally central in bone, so much that biomechanics obviously has to play an important role in the development of bone tissue engineering. We can take advantage of this notion not only by describing the mechanical aspects involved but also by suggesting new solutions to obtain a more effective biomechanical strategy when a bone graft is needed.

\section{Acknowledgements}

The author thanks Dr Alexandre Terrier from the LBO/EPFL for constructive discussions, Salim Darwiche from the LBO/EPFL for English editing of the manuscript and the Inter-Institutional Center for Translational Biomechanics EPFL-CHUV-DAL for the financial support.

\section{References}

Adachi T, Osako Y, Tanaka M, Hojo M, Hollister SJ. 2006. Framework for optimal design of porous scaffold microstructure by computational simulation of bone regeneration. Biomaterials 27:3964-3972.

Aspenberg P. 2009. Bisphosphonates and implants: an overview. Acta Orthop. 80:119-123.

Athanasiou KA, Zhu C, Lanctot DR, Agrawal CM, Wang X. 2000. Fundamentals of biomechanics in tissue engineering of bone. Tissue Eng. 6:361-381.

Behravesh E, Yasko AW, Engel PS, Mikos AG. 1999. Synthetic biodegradable polymers for orthopaedic applications. Clin Orthop. 367S:S118-S129.

Bilodeau K, Mantovani D. 2006. Bioreactors for tissue engineering: focus on mechanical constraints. A comparative review. Tissue Eng. 12:2367-2383.

Blecha LD, Zambelli PY, Ramaniraka NA, Bourban PE, Manson JA, Pioletti DP. 2005. How plate positioning impacts the biomechanics of the open wedge tibial osteotomy; a finite element analysis. Comput Methods Biomech Biomed Eng. 8:307-313.

Blecha LD, Rakotomanana L, Razafimahery F, Terrier A, Pioletti DP. 2009. Targeted mechanical properties for 
optimal fluid motion inside artificial bone substitutes. J Orthop Res. 27:1082-1089.

Brazel E, Taylor D. 2009. Predicting the structural integrity of bone defects repaired using bone graft materials. Comput Methods Biomech Biomed Eng. 12:297-304.

Breuls RG, Sengers BG, Oomens CW, Bouten CV, Baaijens FP. 2002. Predicting local cell deformations in engineered tissue constructs: a multilevel finite element approach. J Biomech Eng. 124:198-207.

Brunski JB. 1991. Influence of biomechanical factors at the bonebiomaterial interface. In: Davis JE, editor. The bonebiomaterial interface. Toronto: University of Toronto Press. p. 391-405.

Buchler P, Pioletti DP, Rakotomanana LR. 2003. Biphasic constitutive laws for biological interface evolution. Biomech Model Mechanobiol. 1:239-249.

Butler DL, Goldstein SA, Guilak F. 2000. Functional tissue engineering: the role of biomechanics. J Biomech Eng. 122:570-575.

Butler DL, Goldstein SA, Guldberg RE, Guo XE, Kamm R, Laurencin CT, McIntire LV, Mow VC, Nerem RM, Sah RL, Soslowsky LJ, Spilker RL, Tranquillo RT. 2009. The impact of biomechanics in tissue engineering and regenerative medicine. Tissue Eng Part B Rev. 15:477-484.

Byrne DP, Lacroix D, Planell JA, Kelly DJ, Prendergast PJ. 2007. Simulation of tissue differentiation in a scaffold as a function of porosity. Young's modulus and dissolution rate: application of mechanobiological models in tissue engineering. Biomaterials. 28:5544-5554.

Carter DR, Fyhrie DP, Whalen RT. 1987. Trabecular bone density and loading history: regulation of connective tissue biology by mechanical energy. J Biomech. 20:785-794.

Carter DR, Beaupre GS, Giori NJ, Helms JA. 1998. Mechanobiology of skeletal regeneration. Clin Orthop Relat Res. 331:S41-S55.

Cavalcant-Adam EA, Shapiro IM, Composto RJ, Macarak EJ, Adams CS. 2002. RGD peptides immobilized on a mechanically deformable surface promote osteoblast differentiation. J Bone Miner Res. 17:2130-2140.

Cowin SC. 2001. Mechanics of materials. In: Cowin SC, editor. Bone mechanics handbook. 2nd ed. Boca Raton: CRC Press. p. $6.1-6.16$.

Datta N, Pham QP, Sharma U, Sikavitsas VI, Jansen JA, Mikos AG. 2006. In vitro generated extracellular matrix and fluid shear stress synergistically enhance 3D osteoblastic differentiation. Proc Natl Acad Sci USA. 103:2488-2493.

Epari DR, Taylor WR, Heller MO, Duda GN. 2006. Mechanical conditions in the initial phase of bone healing. Clin Biomech (Bristol Avon). 21:646-655.

Ghanbari J, Naghdabadi R. 2009. Nonlinear hierarchical multiscale modeling of cortical bone considering its nanoscale microstructure. J Biomech. 42:1560-1565.

Guldberg RE. 2002. Consideration of mechanical factors. Ann NY Acad Sci. 961:312-314.

Han Y, Cowin SC, Schaffler MB, Weinbaum S. 2004. Mechanotransduction and strain amplification in osteocyte cell processes. Proc Natl Acad Sci USA. 101:16689-16694.

Handschel J, Wiesmann HP, Stratmann U, Kleinheinz J, Meyer U, Joos U. 2002. TCP is hardly resorbed and not osteoconductive in a non-loading calvarial model. Biomaterials 23:1689-1695.

Huiskes R, Ruimerman R, van Lenthe GH, Janssen JD. 2000. Effects of mechanical forces on maintenance and adaptation of form in trabecular bone. Nature. 405:704-706.
Humphrey JD. 2001. Stress, strain, and mechanotransduction in cells. J Biomech Eng. 123:638-641.

Ingber DE, Mow VC, Butler D, Niklason L, Huard J, Mao J, Yannas I, Kaplan D, Vunjak-Novakovic G. 2006. Tissue engineering and developmental biology: going biomimetic. Tissue Eng. 12:3265-3283.

Jacobs CR, Yellowley CE, Davis BR, Zhou Z, Cimbala JM, Donahue HJ. 1998. Differential effect of steady versus oscillating flow on bone cells. J Biomech. 31:969-976.

Jaecques SV, Van Oosterwyck H, Muraru L, Van Cleynenbreugel T, De Smet E, Wevers M, Naert I, Vander Sloten J. 2004. Individualised, micro CT-based finite element modelling as a tool for biomechanical analysis related to tissue engineering of bone. Biomaterials. 25:1683-1696.

Jasty M, Bragdon C, Burke D, O'Connor D, Lowenstein J, Harris WH. 1997. In vivo skeletal responses to poroussurfaced implants subjected to small induced motions. J Bone Joint Surg Am. 79:707-714.

Jones AC, Arns CH, Hutmacher DW, Milthorpe BK, Sheppard AP, Knackstedt MA. 2009. The correlation of pore morphology, interconnectivity and physical properties of 3D ceramic scaffolds with bone ingrowth. Biomaterials 30:1440-1451.

Juncosa N, West JR, Galloway MT, Boivin GP, Butler DL. 2003. In vivo forces used to develop design parameters for tissue engineered implants for rabbit patellar tendon repair. J Biomech. 36:483-488.

Jungreuthmayer C, Donahue SW, Jaasma MJ, Al-Munajjed AA, Zanghellini J, Kelly DJ, O'Brien FJ. 2009. A comparative study of shear stresses in collagen-glycosaminoglycan and calcium phosphate scaffolds in bone tissue-engineering bioreactors. Tissue Eng Part A 15:1141-1149.

Kelly DJ, Prendergast PJ. 2006. Prediction of the optimal mechanical properties for a scaffold used in osteochondral defect repair. Tissue Eng. 12:2509-2519.

Klein-Nulend J, Bacabac RG, Mullender MG. 2005. Mechanobiology of bone tissue. Pathol Biol (Paris). 53:576-580.

Lacouture ME, Schaffer JL, Klickstein LB. 2002. A comparison of type I collagen, fibronectin, and vitronectin in supporting adhesion of mechanically strained osteoblasts. J Bone Miner Res. 17:481-492.

Lacroix D, Planell JA, Prendergast PJ. 2009. Computer-aided design and finite-element modelling of biomaterial scaffolds for bone tissue engineering. Philos Trans A Math Phys Eng Sci. 367:1993-2009.

Lenas P, Moos MJ, Luyten F. 2009. Developmental engineering: a new paradigm for the design and manufacturing of cell based products. Part II: From genes to networks: tissue engineering from the viewpoint of systems biology and network science. Tissue Eng Part B Rev. 15:395-422.

Liebschner MA. 2004. Biomechanical considerations of animal models used in tissue engineering of bone. Biomaterials 25:1697-1714.

Lin CY, Kikuchi N, Hollister SJ. 2004. A novel method for biomaterial scaffold internal architecture design to match bone elastic properties with desired porosity. J Biomech. 37:623-636.

Martin I, Smith T, Wendt D. 2009. Bioreactor-based roadmap for the translation of tissue engineering strategies into clinical products. Trends Biotechnol. 27:495-502.

Mathieu LM, Montjovent MO, Bourban PE, Pioletti DP, Manson JA. 2005. Bioresorbable composites prepared by supercritical fluid foaming. J Biomed Mater Res. 75:89-97.

Mathieu LM, Mueller TL, Bourban PE, Pioletti DP, Muller R, Manson JA. 2006. Architecture and properties of anisotropic 
polymer composite scaffolds for bone tissue engineering. Biomaterials. 27:905-916.

Mauney JR, Sjostorm S, Blumberg J, Horan R, O'Leary JP, Vunjak-Novakovic G, Volloch V, Kaplan DL. 2004. Mechanical stimulation promotes osteogenic differentiation of human bone marrow stromal cells on 3-D partially demineralized bone scaffolds in vitro. Calcif Tissue Int. 74:458-468.

Meinel L, Karageorgiou V, Fajardo R, Snyder B, Shinde-Patil V, Zichner L, Kaplan D, Langer R, Vunjak-Novakovic G. 2004. Bone tissue engineering using human mesenchymal stem cells: effects of scaffold material and medium flow. Ann Biomed Eng. 32:112-122.

Meyer U, Joos U, Wiesmann HP. 2004. Biological and biophysical principles in extracorporal bone tissue engineering. Part III. Int J Oral Maxillofac Surg. 33:635-641.

Milan JL, Planell JA, Lacroix D. 2009. Computational modelling of the mechanical environment of osteogenesis within a polylactic acid-calcium phosphate glass scaffold. Biomaterials. 30:4219-4226.

Peter B, Gauthier O, Laib S, Bujoli B, Guicheux J, Janvier P, van Lenthe GH, Muller R, Zambelli PY, Bouler JM et al. 2006. Local delivery of bisphosphonate from coated orthopedic implants increases implants mechanical stability in osteoporotic rats. J Biomed Mater Res. 76:133-143.

Pfeiler TW, Sumanasinghe RD, Loboa EG. 2008. Finite element modeling of 3D human mesenchymal stem cell-seeded collagen matrices exposed to tensile strain. J Biomech. 41:2289-2296.

Pierre J, Oddou C. 2007. Engineered bone culture in a perfusion bioreactor: a 2D computational study of stationary mass and momentum transport. Comput Methods Biomech Biomed Eng. 10:429-438.

Pioletti DP, Rakotomanana LR. 2000. On the independence of time and strain effects in the stress relaxation of ligaments and tendons. J Biomech. 33:1729-1732.

Porter BD, Oldham JB, He SL, Zobitz ME, Payne RG, An KN, Currier BL, Mikos AG, Yaszemski MJ. 2000. Mechanical properties of a biodegradable bone regeneration scaffold. J Biomech Eng. 122:286-288.

Prendergast PJ, Huiskes R, Soballe K. 1997. ESB Research Award 1996. Biophysical stimuli on cells during tissue differentiation at implant interfaces. $\mathrm{J}$ Biomech. 30:539-548.

Sandino C, Planell JA, Lacroix D. 2008. A finite element study of mechanical stimuli in scaffolds for bone tissue engineering. J Biomech. 41:1005-1014.

Sanz-Herrera JA, Garia-Aznar JM, Doblare M. 2008. Micromacro numerical modelling of bone regeneration in tissue engineering. Comput Method Appl M. 197(33-40): 3092-3107.

Sengers BG, Taylor M, Please CP, Oreffo RO. 2007. Computational modelling of cell spreading and tissue regeneration in porous scaffolds. Biomaterials 28:1926-1940.
Sikavitsas VI, Temenoff JS, Mikos AG. 2001. Biomaterials and bone mechanotransduction. Biomaterials 22:2581-2593.

Silber JS, Anderson DG, Daffner SD, Brislin BT, Leland JM, Hilibrand AS, Vaccaro AR, Albert TJ. 2003. Donor site morbidity after anterior iliac crest bone harvest for singlelevel anterior cervical discectomy and fusion. Spine 28:134-139.

Singh H, Teoh SH, Low HT, Hutmacher DW. 2005. Flow modelling within a scaffold under the influence of uni-axial and bi-axial bioreactor rotation. J Biotechnol. 119:181-196.

Srinivasan S, Gross TS. 2000. Canalicular fluid flow induced by bending of a long bone. Med Eng Phys. 22:127-133.

Stadelmann VA, Terrier A, Pioletti DP. 2008. Microstimulation at the bone-implant interface upregulates osteoclast activation pathways. Bone. 42:358-364.

Stevens MM, Marini RP, Schaefer D, Aronson J, Langer R, Shastri VP. 2005. In vivo engineering of organs: the bone bioreactor. Proc Natl Acad Sci USA. 102:11450-11455.

Stiehler M, Bunger C, Baatrup A, Lind M, Kassem M, Mygind T. 2009. Effect of dynamic 3-D culture on proliferation, distribution, and osteogenic differentiation of human mesenchymal stem cells. J Biomed Mater Res. 89:96-107.

Tate ML, Knothe U. 2000. An ex vivo model to study transport processes and fluid flow in loaded bone. J Biomech. 33:247-254.

Terrier A, Sedighi-Gilani M, Roshan Ghias A, Aschwanden L, Pioletti DP. 2009. Biomechanical evaluation of porous biodegradable scaffolds for revision knee arthroplasty. Comput Methods Biomech Biomed Eng. 12:333-339.

Wettergreen MA, Bucklen BS, Sun W, Liebschner MA. 2005. Computer-aided tissue engineering of a human vertebral body. Ann Biomed Eng. 33:1333-1343.

Wiesmann HP, Joos U, Meyer U. 2004. Biological and biophysical principles in extracorporal bone tissue engineering. Part II. Int J Oral Maxillofac Surg. 33:523-530.

Williams JM, Adewunmi A, Schek RM, Flanagan CL, Krebsbach PH, Feinberg SE, Hollister SJ, Das S. 2005. Bone tissue engineering using polycaprolactone scaffolds fabricated via selective laser sintering. Biomaterials 26:4817-4827.

Wood MA, Yang Y, Thomas PB, Haj AJ. 2006. Using dihydropyridine-release strategies to enhance load effects in engineered human bone constructs. Tissue Eng. 12:2489-2497.

Yu X, Botchwey EA, Levine EM, Pollack SR, Laurencin CT. 2004. Bioreactor-based bone tissue engineering: the influence of dynamic flow on osteoblast phenotypic expression and matrix mineralization. Proc Natl Acad Sci USA. 101:11203-11208.

Zhang ZY, Teoh SH, Chong WS, Foo TT, Chng YC, Choolani M, Chan J. 2009. A biaxial rotating bioreactor for the culture of fetal mesenchymal stem cells for bone tissue engineering. Biomaterials 30:2694-2704. 